\title{
Editorial: Energy Dissipation Systems and Seismic Fuses
}

\author{
Farhad Behnamfar* \\ Department of Civil Engineering, Isfahan University of Technology, Isfahan, Iran
}

Keywords: energy dissipation, seismic fuse, damage, safety, replaceable

Editorial on the Research Topic

Energy Dissipation Systems and Seismic Fuses

It has become common knowledge in the earthquake engineering community that proper design of a structure to withstand seismic forces in a highly seismic prone area is not possible without accepting a certain level of damage. That being said, it is essential to explore and establish a rational basis for defining the level of acceptable damage and the location where the damage can emerge and develop. Nowadays, the notion of seismic fuses has found its place within this field as it has been accepted large and wide as a practical solution for safe design. A seismic fuse is introduced as a part of the lateral load bearing system that envelops the earthquake damage in a stable manner while protecting the other parts and leaving them intact. They are installed intentionally to be easily replaceable if not repaired. Research on this topic is ongoing and innovative, bringing together engineering and art.

It was the main incentive for bringing up the research topic "Energy Dissipation Systems and Seismic Fuses".

Under this topic, the main aim was to foster the idea of localizing the seismic damage, while at the same time protecting the other structural members and the nonstructural elements. As made clear through the extensive research in this area, it is a feasible solution for various structures. The problem is that most of the research tasks have been concentrated on the energy dissipation device itself, not on its performance in the whole structural system. With that in mind, studying both in-plane and out-of-plane behavior of the seismic fuses when mounted in a system it is intended for, is at stake when examining the action of earthquakes.

Papers outlining the research works on proposing and developing new practical ideas for earthquake energy dissipation systems and devices acting as seismic fuses were invited to be submitted under the mentioned topic. The suggested research themes were enumerated to be Innovative seismic fuses for different types of structural systems and building materials, description of various types of testing protocols for seismic energy dissipation devices, experimental evaluation of seismic fuse performance in structural prototypes, real performance of energy dissipation devices as observed in the recent large earthquakes, comparative works on the efficiency and effectiveness of novel seismic fuses, developing design recommendations for various types of seismic energy dissipation devices.

In response to the invitation, 25 positive responses for potentially sending contributions were received. Afterwards, eight abstracts were submitted for review and accepted provisionally. At the stage of full paper submission, four papers were received from the corresponding authors, out of which 3 papers were accepted.

The accepted papers targeted highly active trends under the research topic. In the paper entitled "Seismic Performance of Inerter and Negative Stiffness-Based Dampers for Vibration Control of Structures" (Isalm and Jangid), performance of the negative stiffness and inerter based energy dissipation devices were evaluated as possible alternatives to traditional energy dissipation devices. The mathematical formulation and optimal design of some novel negative stiffness and inerter based mechanisms were presented. A 20-story building was analyzed as a case study structure. Efficiency of 
the proposed devices in reducing the inter-storey drifts and floor accelerations was demonstrated in comparison to other existing damping induced components.

The second paper was entitled "Earthquake Damage Reduction in Timber Frame Houses Using Small-Size Fluid Damper" (Nakamura and Fuji). In the mentioned research work, application of small fluid dampers for seismic damage reduction in timber frame houses was studied numerically. Detailed analytical model of an actual two-story timber structure house was analyzed under the 2016 Kumamoto earthquake for this purpose. The small-size fluid damper was equipped with a relief mechanism for the damping force. Effects of installing four, and then seven fluid dampers in the 1st story of the model on the earthquake damage reduction were explored. It was shown that the four and seven fluid damper alternatives reduce the maximum 1st-story drift angle by one-third and half, respectively.

In the paper entitled "Seismic design and performances of frame structures connected to a strongback system and equipped with different arrangements of supplemental viscous dampers" (Palermo et al.), the authors conducted a study on the seismic behavior of a moment-resisting frame structure supported by a vertical rigid truss, known as "strongback," equipped with added fluid-viscous dampers. The strongback truss was designed to remain in the elastic range under strong seismic ground motion. The supplemental dampers were inserted to dissipate a considerable amount of seismic energy. In the study, analytical formulations were provided for the preliminary design of the added dampers, and to evaluate the increase in energy dissipation capability for the selected dampers configurations. The formulas were developed for different configurations of the added viscous dampers including the dampers inserted within the frame between consecutive stories as an inter-storey placement, and dampers located at the base of the strongback to realize a rigid dissipative tower. The effectiveness of the dampers configurations was evaluated through dynamic analyses.

In conclusion, it is hoped that this forum has been successful in its mission to help foster the development of the energy dissipation systems and seismic fuses as a means for constructing safer and more robust buildings in seismic prone areas of the world.

\section{AUTHOR CONTRIBUTIONS}

The author confirms being the sole contributor of this work and has approved it for publication.

Conflict of Interest: The author declares that the research was conducted in the absence of any commercial or financial relationships that could be construed as a potential conflict of interest.

Publisher's Note: All claims expressed in this article are solely those of the authors and do not necessarily represent those of their affiliated organizations, or those of the publisher, the editors and the reviewers. Any product that may be evaluated in this article, or claim that may be made by its manufacturer, is not guaranteed or endorsed by the publisher.

Copyright (c) 2022 Behnamfar. This is an open-access article distributed under the terms of the Creative Commons Attribution License (CC BY). The use, distribution or reproduction in other forums is permitted, provided the original author(s) and the copyright owner(s) are credited and that the original publication in this journal is cited, in accordance with accepted academic practice. No use, distribution or reproduction is permitted which does not comply with these terms. 